\title{
On the Analysis Performance of Updating Weight for Estimation Target of Drone System
}

\author{
Kwan Hyeong Lee ${ }^{1}$ and Jae Hoon Lee ${ }^{2}$ \\ ${ }^{1}$ Devisino of Human IT Convergence, 1007 University of Daejin, South Korea \\ ${ }^{2}$ Devision of Human-Architectural Engineering, 1007 University of Daejin, South Korea
}

\begin{abstract}
In this paper, we propose the method which desired signal is estimated by updating the weight of the MVDR algorithm. The MUSIC algorithm is generally a lot of used in the direction of arrival estimation method. The MUSIC algorithm has a good resolution because of using subspace techniques consisting of a signal subspace and a noise subspace. The processor capability of drone system is required low power consumption and low computation complexity because it uses a microprocessor. If the drone system has a lot of computation complexity, the desired signal cannot be estimated. This paper study a method estimating the desired signal with a simple calculation. The proposed method is updated weight by the covariance matrix of MVDR algorithm. Through simulation, we analyse performance by comparing MVDR, MUSIC and the proposed method. In the simulation results, the proposed method is the same as the MUSIC algorithm in direction of arrival estimation. Since the proposed method has no subspace, it reduces computational complexity than MUSIC algorithm. The desired signal estimation of the proposed method is superior to the MVDR algorithm.
\end{abstract}

\section{Introduction}

Recently, the estimation of direction of arrival(DoA) for target has been many studied according to the development of wireless communication technique. Direction of estimation method have been widely used in many applications such as radar, sonar, biomedical, and communication systems. DoA estimation methods are Bartlett, Capon, Linear predict, MUSIC, and ESPRIT [13]. Also, the estimation method of target is divided into two methods. First, there is non-parameter method such as Bartlett and Capon, finally, there is parameter method such as MUSIC and ESPRIT. MUSIC estimation method has a super resolution because it uses the subspace method. There has a lot of computation complexity because the MUSIC method uses eigenvalue decomposition. In order to improve the DoA estimation, there are improved signal to noise ratio, higher transmission power, and adaptive array signal processing [4-6].

In this paper, we propose the method which is a low computation and an accurate DoA estimation. The proposed method is estimating the desired signal by updating the weight of the MVDR (Minimum Variance Distortionless Response) algorithm. Generally, the MVDR algorithm is called Capon method. The Capon method has poor resolution to estimate the desired signal due to low computation complexity and inaccurate weight. We propose a method how to improve the weight of MVDR algorithm. The proposed method for improving the weight use an adaptive array antenna and a beamforming technique. The adaptive array method finds the covariance matrix using Lagrange multiplier and applies the adaptive array algorithm to improve the resolution. And, we divided it into two step to find optimum weight. The source signal covariance matrix is obtained in two steps of the proposed method. As a result, we can obtain the covariance matrix of the received signals. The MUSIC method is mostly used to estimate for target in spatial. But, it is not effective for drone system to use the MUSIC method because of the processing capability of microprocessor. The processing capability of drone system using microprocessor degrades form computation complexity and power consumption, so that it cannot accurately estimate the desired signal.

The organization of this paper is as follow. In section 2 , the signal mode analysis considered is described. The output power of MVDR algorithm and the proposed weight of covariance matrix are presented in Section 3. A performance analysis of the proposed method is provided in Section 4. Conclusions are drawn in Section 5.

\section{Signal model analysis}

Figure 1 shows an adaptive array system. We consider that the receiver is uniform linear array composed of an $\mathrm{M}$-arrays antenna with adjacent array element spacing $\mathrm{d}$ deployed at the figure 1 and $\mathrm{N}$ narrowband signals. The $\mathrm{N}$-dimensional column vector $\mathrm{a}\left(\theta_{K}\right)$, the antenna array response vector is as follow[7-8]

$$
\mathrm{a}\left(\theta_{k}\right)=\left[1, a\left(\theta_{1}\right), \cdots, a\left(\theta_{K}\right)\right]^{T}
$$


Where $\mathrm{a}\left(\theta_{k}\right)=\exp \left(-\frac{\mathrm{j} 2 \pi \mathrm{d} \cos \theta_{k}}{\lambda}\right)$. Bothd $\lambda$ and $k$ are the the weavelenght and incident signal on array antenna, respectively. Then, output signal of the array antenna is as follow

$$
X(t)=A s(t)+N(t)
$$

$X(t)$ : the incident signal of the array antenna.

$s(t)$ : the source signal of the array antenna.

$N(t)$ : the noise signal of the array antenna.

$A=\left[a\left(\theta_{1}\right), a\left(\theta_{2}\right), \cdots, a\left(\theta_{K}\right)\right]:$ steering matrix

Where $a(\theta)$ is steering vector corresponding to the direction of arrival of $k$ th signal. The covariance matrix of the received signal vector on the array antenna can be written as follow

$$
R=E\left[X(t) X^{H}(t)\right]=\frac{1}{L} \sum_{k=1}^{N} X(t) X^{H}(t)
$$

Where $L$ is a number of snapshot.

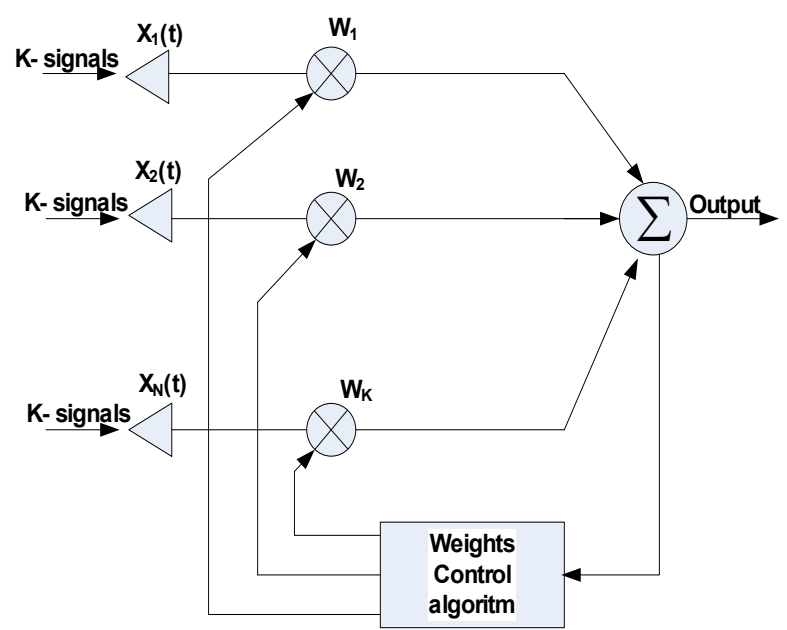

Fig 1. Block diagram of the adaptive array system.

\section{Covariance matrix of estimation signal}

\subsection{Output spectrum of MVDR Algorithm}

In this chapter, we discuss the MVDR algorithm for estimating the direction of arrival. Output signal of incident wave at receiver can be written as follow[9-11]

$$
X(t)=x(t)+N(t)
$$

where, $x(t)=s(t) a(\theta) . a(\theta)$ and $s(t)$ is called the array steering vector and the source signals on the array antenna, respectively. $N(t)$ is a zero mean complex Gaussian random signal $\left(\sigma^{2} I\right) . \sigma$ and $I$ are a variance and unit matrix, respectively. Output signal is represented by multiplier of weight(W) and the receiver signal on array element. When the weight vector is $1 \mathrm{x} \mathrm{N}$, output signal to minimize the variance of $Y(t)$ in the noise can be written as follow

$$
Y_{n}=W^{H} X(t)
$$

In order to minimize output noise $\mathrm{E}\left[\left|Y_{n}\right|^{2}\right]$, the constraint of no distortion can be written as follow

$$
Y_{n}=W\left(A R_{s} \mathrm{~A}^{H}+\sigma^{2} I\right) W^{H}
$$

Where $R_{s}=E\left[s(t) s^{H}(t)\right]$ is a source signal correlation matrix. The constraint condition is as follow

$$
W^{H} a(\theta)=1
$$

The output noise power is can be as follow

$$
E\left[\left|Y_{n}\right|^{2}\right]=W^{H} E\left[X(t) X^{H}(t)\right] W
$$

Where ()$^{H}$ is Hermit matrix. We would like to minimize the output noise power in subject to the constraint at the equation (7). The output power is as follow

$$
E[Y(t)]=E[X(t)]+E[N(t)]=E[s(t)]
$$

Let us assume that

$$
\begin{gathered}
E\left[N(t) N^{*}(t)\right]=\left[\begin{array}{cccc}
\sigma_{1} & 0 & \cdots & 0 \\
0 & \sigma_{N} & \cdots & 0 \\
\vdots & \vdots & \ddots & \vdots \\
0 & \cdots & \cdots & \sigma_{N}
\end{array}\right] \\
R=E\left[X(t) X^{*}(t)\right]=\sum_{k=1}^{N} s_{k} a_{k} a_{k}^{*}+\left[\begin{array}{cccc}
\sigma_{1} & 0 & \cdots & 0 \\
0 & \sigma_{N} & \cdots & 0 \\
\vdots & \vdots & \ddots & \vdots \\
0 & \cdots & \cdots & \sigma_{N}
\end{array}\right] \\
=\left[a_{1}, \cdots, a_{N}\right]\left[\begin{array}{cccccc}
s_{1} & 0 & 0 & \cdots & \cdots & 0 \\
0 & \ddots & \cdots & 0 & 0 & 0 \\
0 & 0 & s_{N} & \cdots & \cdots & 0 \\
0 & \vdots & \vdots & \sigma_{1} & \vdots & 0 \\
0 & 0 & \cdots & \cdots & \ddots & 0 \\
0 & 0 & \cdots & 0 & \cdots & \sigma_{N}
\end{array}\right]\left[\begin{array}{c}
a_{1}^{*} \\
a_{2}^{*} \\
\vdots \\
a_{N}^{*}
\end{array}\right] \quad(12)
\end{gathered}
$$

We find it the solution minimizing the variance of the output signal by using a Lagrange multiplier. The solution can be as follow

$$
\begin{array}{r}
S \triangleq W^{H}(t) R W+\lambda(t)\left[W^{H} a(\theta)-1\right]+ \\
\lambda^{*}(t)[a(\theta) W-1]
\end{array}
$$

Taking the gradient with respect to $W^{H}$ in equation(14) as follow

$$
W_{o p}^{H}=-\lambda(t) a^{H}(\Theta) R^{-1}
$$

Substituting equation(13) into equation(7) to find $\lambda(t)$, which give by

$$
\lambda(t)=-\left[a^{H}(\theta) R^{-1}(t) a(\theta)\right]^{-1}
$$

Thus, optimum weight can be written as follow

$$
W_{o p}^{H}(t)=B(\theta) a^{H}(\theta) R^{-1}
$$

Where

$$
B(\theta)=\left[a^{H}(\theta) R^{-1} a(\theta)\right]^{-1}
$$


The equeation(16) is called the MVDR algorithm. Array output power is as follow

$$
P_{M V D R}=\frac{1}{a^{H}(\theta) R^{-1} a(\theta)}
$$

\subsection{Proposed covariance matrix in mutual coupling}

We consider that all signals of the receiving on array antennas are coherent. Source signal of each array antenna are amplitude and phase delayed due to multipath. First array antenna is reference signal. In the case of $s_{k}(k=$ $1,2, \cdots, K)$ narrowband sources, Replicas of the first array antenna source signal can be as follow

$$
s_{k}(t)=h s_{1}(t)
$$

Where $h$ represents the complex attenuation of the $k$ th signal with respect to the first signal. The signal correlation matrix is as follow

$$
R_{S}=H H^{H}
$$

Where $H=\left[h_{1}, h_{2}, \cdots, h_{K}\right]^{T}$. We have to remove the effects of mutual coupling before estimating desired signals because it is impossible to estimate desired direction of arrival signals. We have the following steps:

Step 1. eigenvalue decomposition of $R_{S}$ can be written as follow

$$
R_{s}=u_{s} \lambda_{s} u_{s}^{H}+u_{c} \lambda_{c} u_{c}^{H}
$$

Where $u$ is $M \times 1$ eigenvector corresponding to the largest eigenvalue, and $u$ is eigenvector corresponding to the smallest eigenvalue by $M-1$. According to subspace method, we have as follow

$$
A \cdot H=b C^{-1} u_{s} \perp \operatorname{span}\left\{C^{-1} u_{c}\right\}
$$

Where $b$ and $C$ are constant and mutual coupling matrix, respectivly. Mutual coupling matrix is as follow

$$
\mathrm{C}=\left[\begin{array}{cccccc}
1 & c_{1} & \cdots & c_{m} & \cdots & 0 \\
c_{1} & 1 & c_{1} & \cdots & \ddots & 0 \\
\vdots & c_{1} & 1 & \ddots & \cdots & c_{m} \\
c_{m} & \cdots & \ddots & \ddots & c_{1} & \vdots \\
0 & \ddots & \cdots & \ddots & 1 & c_{1} \\
0 & 0 & c_{m} & \cdots & c_{1} & 1
\end{array}\right]
$$

Step 2. Reconstruct the covariance matrix of the array output is as follow

$$
R_{s}=b C^{-1} u_{s} u_{s}^{H} C^{H} b^{H}
$$

Spatial smoothing is represented from subarray method which the nature array is divided uniform overlapping subarray.

\section{Simulation \& performance analysis}

In this chapter, we analyse the performance to compare classical direction of arrival method with the proposed method. The classical method used which is MVDR and MUSCI. With the simulation condition, Snapshot, SNR, and targets were 100times, $10 \mathrm{~dB}$, and objects 2, respectively. Figure 2 shows the angle estimated by the MVDR algorithm using 9 array elements, and the desired signal estimate at $\left(-20^{\circ}, 20^{\circ}\right)$. In figure 2 , the desired signal of two targets is estimated accurately at $\left(-20^{\circ}, 20^{\circ}\right)$. Figure 3 shows the angle estimated by the MVDR algorithm with 6 array elements, and the desired signal estimate at $\left(-5^{\circ}\right.$, $5^{\circ}$ ) as two targets. In figure 3 , the desired signals could not be estimated accurately at $\left(-5^{\circ}, 5^{\circ}\right)$. The desired signal estimation in figure 3 represent one signal at $\left(0^{\circ}\right)$. Figure 4 shows the angle estimated by the MUSIC algorithm with 6 array elements, and the desired signal estimate at $\left(-5^{\circ}, 5^{\circ}\right)$. In figure 4 , the desired signals of two targets could were estimated accurately at $\left(-5^{\circ}, 5^{\circ}\right)$. Figure 5 shows the angle estimated by the proposed method with 6 array elements, and the desired signal estimate at $\left(-5^{\circ}, 5^{\circ}\right)$. In figure 5 , the desired signals of two targets were estimated accurately at $\left(-5^{\circ}, 5^{\circ}\right)$.

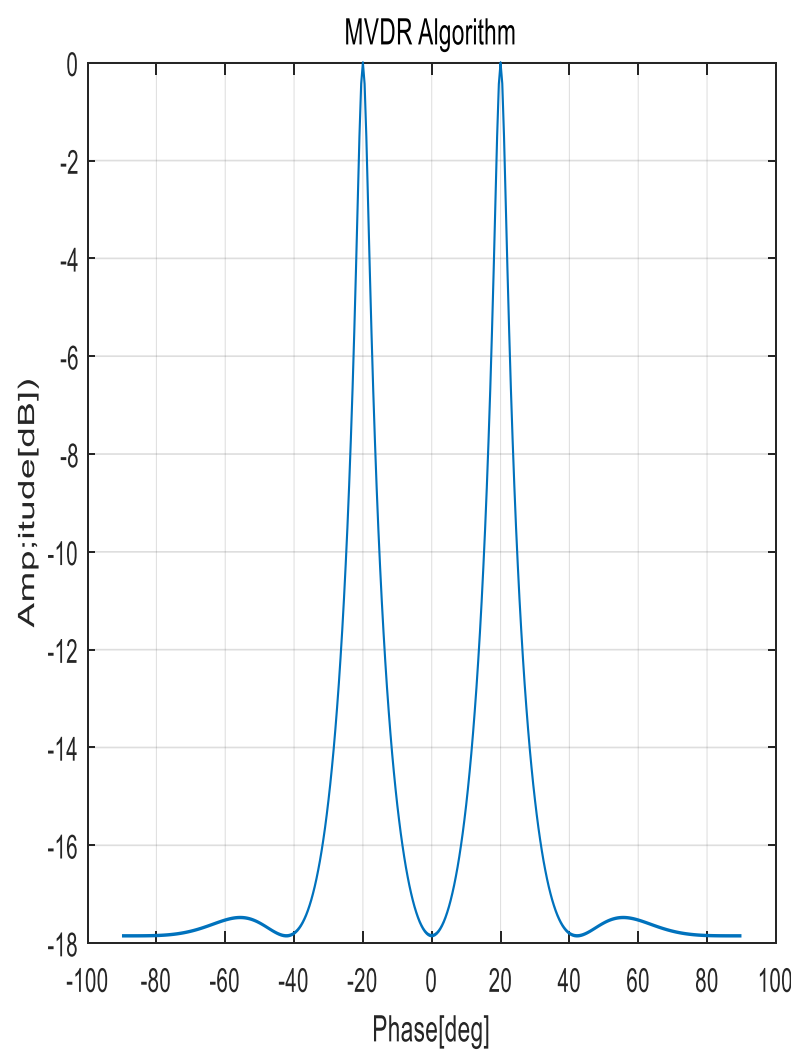

Fig 2. Angle of estimation $\left(-20^{\circ}, 20^{\circ}\right)$ of the MVDR algorithm $\mathrm{SNR}=10 \mathrm{~dB}$, array element $=9$ 


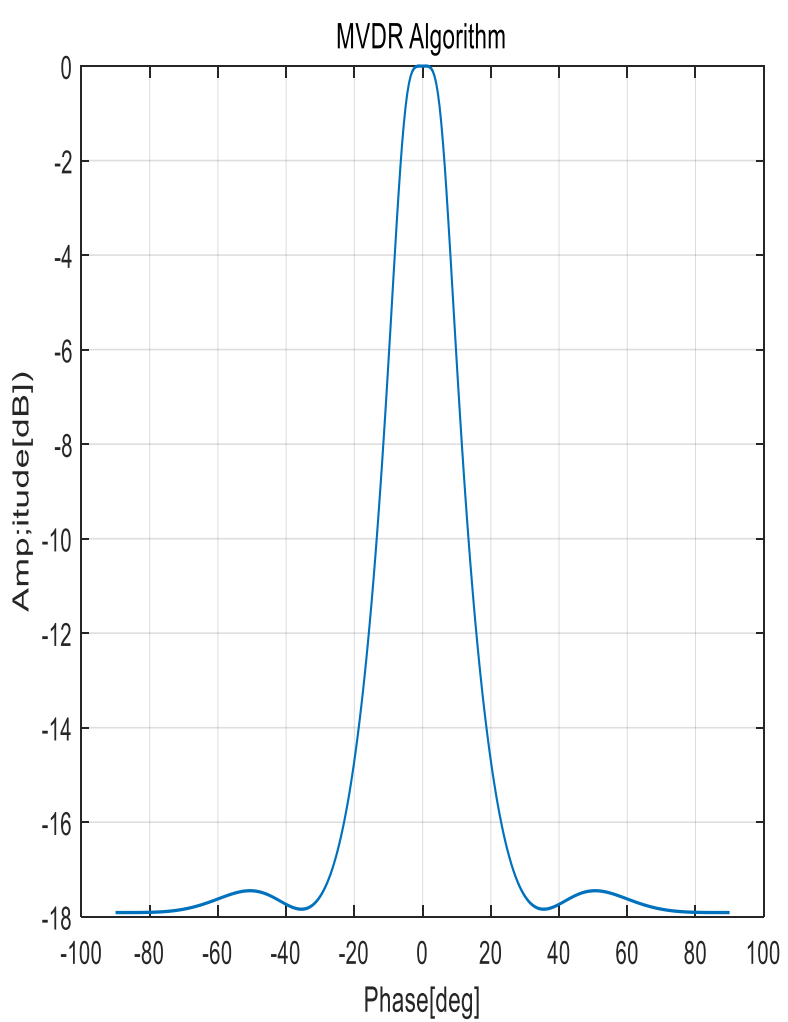

Fig 3. Angle of estimation $\left(-5^{\circ}, 5^{\circ}\right)$ of the MVDR algorithm, $\mathrm{SNR}=10 \mathrm{~dB}$, array element $=6$

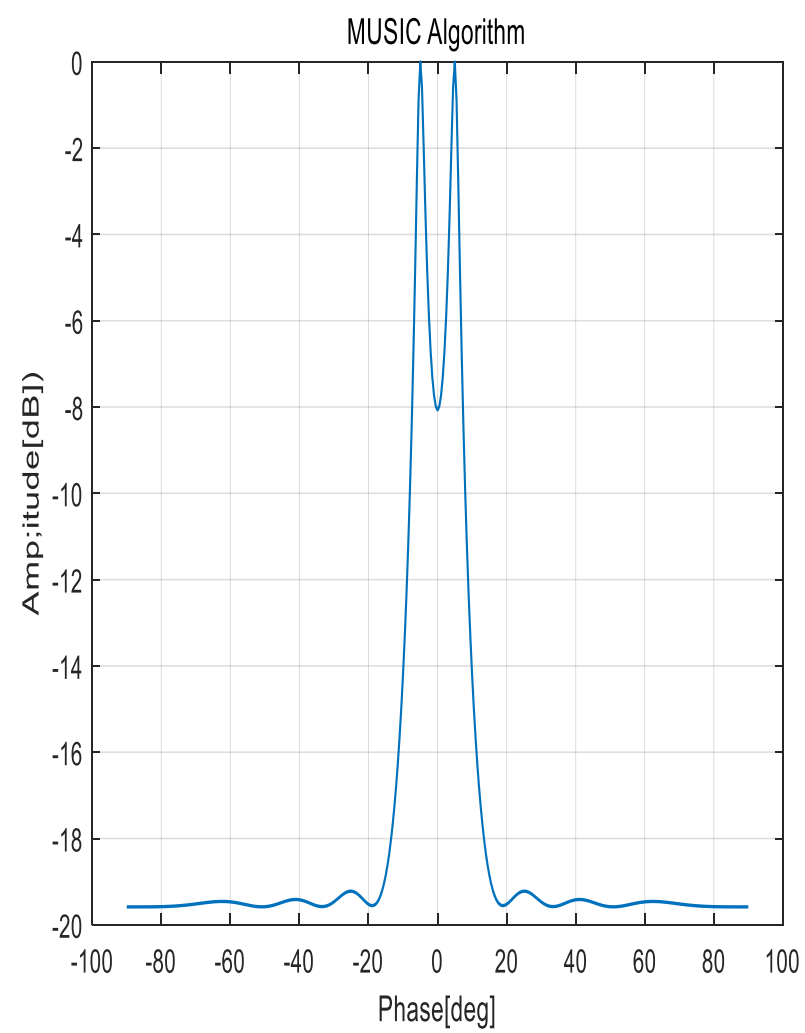

Fig 4. Angle of estimation $\left(-5^{\circ}, 5^{\circ}\right)$ of the MUSIC algorithm, $\mathrm{SNR}=10 \mathrm{~dB}$, array element $=6$

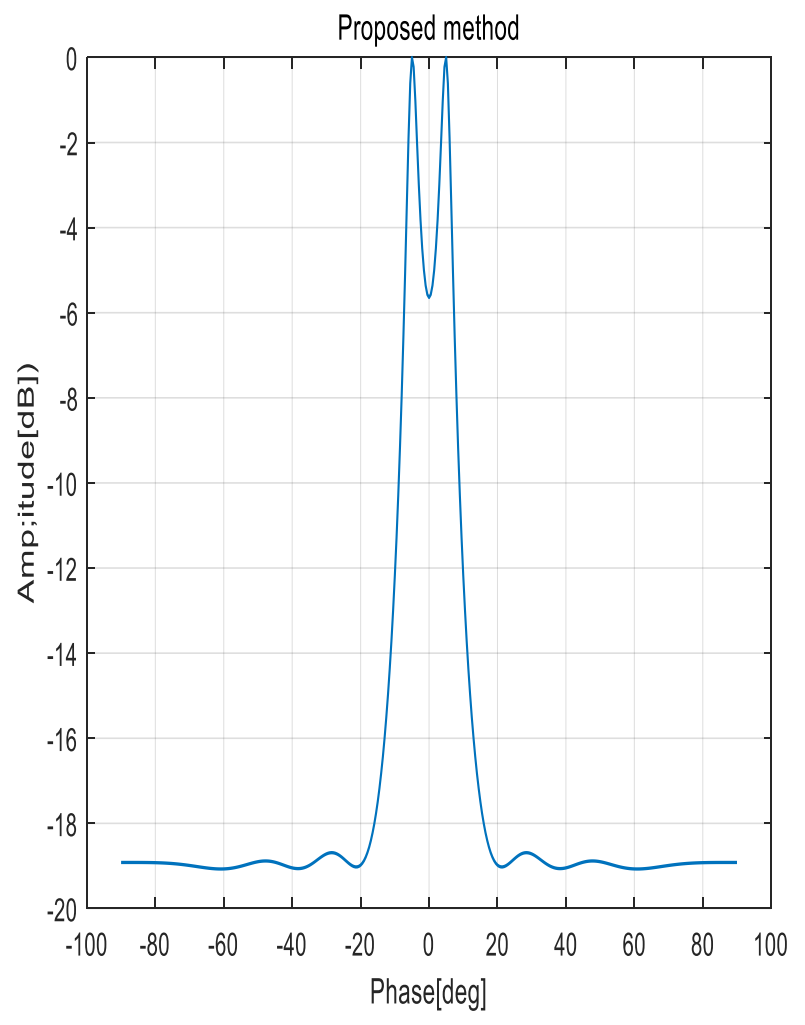

Fig 5. Angle of estimation $\left(-5^{\circ}, 5^{\circ}\right)$ of the proposed algorithm, $\mathrm{SNR}=10 \mathrm{~dB}$, array element $=6$

\section{Conclusion}

In this paper, we studied the proposed method to accurately estimation the desired signal with the modified MVDR algorithm. The proposed method is to estimate the direction of arrival by updated weight. First, we studied the MVDR algorithm to obtain the weight. Second, the obtained weight is updated by covariance matrix. Final, the covariance matrix is obtained by the mutual coupling matrix of Toeplitz matrix. In simulation, the classical MVDR algorithm have a poor resolution. Comparing figure 4 and figure 5, the resolution of the proposed method is the same as the MUSIC algorithm. The proposed method has much better resolution than the convention MVDR algorithm. Since the proposed has no subspace, the calculation complexity decreases than the MUSIC algorithm. The proposed method is suitable for the drone system using microprocessors to detection others targets.

\section{Acknowledgment}

This work was supported by the National Research Foundation of Korea grant funded by the Korea government (NRF-2017R1D1A1B03032556).

\section{References}

1. Harry L. Van Trees, Optimum array processing (John Wiley\&Sons, 2002)

2. S. Unnikrishna Pillai, Array signal processing (Springer-Verlag, 1989) 
3. Frank B. Gross, Smart antenna for wireless communications with matlab (McGraw-Hill,2005)

4. B. Allen ,M.Ghavami, Adaptive array systems (John Wiley\&Sons, 2005)

5. Don H.Johanson, Dan E. Dudgeon, Array signal processing (Prentice-Hall, 1963)

6. Mark C. Sullivan Practical array processing (McGraw-Hill 2009)

7. Youssef. Khmou, Said. Safi, Miloud. Frike, Comparative Study between Several Direction of Arrival Estimation Methods, Journal of Telecommunication and Information Technology, 1,41 (2014)
8. Khairy A. El barbary, Tawil S. Mohamed, Mohamed.S.Melad, High Resolution Direction of Arrival Esitmation(Coherent Signal Source DOA Estiamtioin), IJERA, 3, 132 (2013)

9. Petre Stoica, Prabhu Babu, Jian Li, SPICE: ASparse Convariance-Based Esitmation Method for Array Processing, IEEE Trans. SP, 59, 629 (2011)

10. Liran Tzafri, Anthony j, Weiss, High-Resolution Direct Position Determination using MVDR, IEEE Trans. WC, 15, 6449 (2016)

11. Ying Liu, Chi Xie, Yourun Zhang, Direction of arrivals estimation for correlated broadband radio signals by MVDR algorithm using wavelet, IEEE JM, 190 (2017) 